\title{
Recurrent ectopic pregnancy after partial salpingectomy, distal and proximal, therapeutic and prophylactic
}

\author{
Sharif I. M. F. Ismail
}

Received: 11 March 2008 /Accepted: 13 June 2008 / Published online: 23 July 2008

(C) Springer-Verlag 2008

\begin{abstract}
Salpingectomy should always be complete. A patient had a third ectopic pregnancy following partial salpingectomy, of the ampullary and uterine parts, therapeutically and prophylactically.
\end{abstract}

Keywords Partial salpingectomy Prophylactic $\cdot$ Recurrent ectopic $\cdot$ Repeat $\cdot$ Therapeutic

\section{Case}

A 37-year-old patient was seen in the early pregnancy clinic with left iliac fossa pain after 6 weeks of amenorrhea. Pregnancy test was positive and transvaginal ultrasound scan showed an empty uterus with a thickened endometrium, a normal right ovary and a prominent left ovary, containing a solid echo-bright area and a bleeding ovarian cyst was suggested. Her $\beta$-human chorionic gonadotropin (HCG) level was estimated to be $1,283 \mathrm{u} / 1$ and rose to $1,867 \mathrm{u} / 13$ days later.

Earlier, the patient had a forceps delivery at term followed by a ruptured right tubal ectopic, that was managed by open right salpingectomy, where the ampullary part of the tube was excised. This was followed by a ruptured right tubal ectopic pregnancy in the residual stump.

This was managed by removing what was left of the right tube through another laparotomy. Although the left tube was found to be unhealthy, a left partial salpingectomy

\footnotetext{
S. I. M. F. Ismail ( $\square)$

Department of Obstetrics and Gynaecology,

Yeovil District Hospital,

Yeovil BA21 4AT,

Somerset, England, UK

e-mail: sharif212121@yahoo.co.uk
}

was carried out, removing the uterine part, to avoid future ectopic.

In view of the rising $\beta-H C G$ levels and the previous history of recurrent ectopic pregnancy, a diagnostic laparoscopy was advised and arranged. Blood was seen in the pouch of Douglas and the utero-vesical pouch and omental adhesions were noted below the scar of the previous two laparotomies.

The adhesions were divided using unipolar diathermy and suction irrigation was carried out to enable accurate examination of the pelvis. The right ovary was normal. The left ovary was tangled in a haemorrhagic mass that included ruptured ectopic pregnancy in the residual left tube. Further suction irrigation was carried out to enable separating the ovary from the remaining part of the tube, which was then removed using two endo-loops. The patient made an uneventful recovery and was discharged home a few hours later.

\section{Discussion}

The choice between salpingectomy and salpingostomy revolves about the likelihood of subsequent live intrauterine pregnancy, persistent trophoblastic disease and recurrence of ectopic pregnancy [1]. Salpingectomy has long been known to be followed by less frequent recurrent ectopic pregnancy that salpingostomy [2]. The principal advantage in resorting to salpingectomy is to avoid recurrent ectopic pregnancy in an unhealthy tube. As the incidence of such recurrence rises with the number of ectopic pregnancies [3], it is logical that salpingectomy is the right choice following a recurrent ectopic pregnancy.

In the case described here, the patient had recurrent ectopic pregnancy twice despite having partial salpingec- 
tomy in the respective tubes, one at a time. The benefit of opting for such an excisional approach, in comparison to salpingostomy was not achieved, defeating the argument for taking such a line. In fact, the patient had prophylactic left partial salpingectomy without there being an ectopic during her second laparotomy, only to have an ectopic in the residual part of that tube 6 years later. It is possible that this tube was damaged and the patient would have had an ectopic pregnancy if it were to have been left over. It is also possible however, at least in theory, that the partial salpingectomy led to tubal narrowing and contributed to the ectopic pregnancy developing in the residual part of the tube. On the basis of this line of thinking, the prophylactic left partial salpingectomy was not only unnecessary, but rather harmful.

Partial salpingectomy entails excision of part of the tube, leaving a portion behind. This can be carried out either laparoscopically or through a laparotomy. Several reports have described recurrent ectopic pregnancy after partial salpingectomy, both open and laparoscopic, where uterine and ampullary parts were removed $[4,5]$.

Bilateral ectopic pregnancy has been reported after bilateral partial salpingectomy for contraception [6]. This is the first case report describing ectopic pregnancy after prophylactic partial salpingectomy and the first one where ectopic followed partial salpingectomy twice, both of ampullary and uterine parts, therapeutic and prophylactic.

These cases should call for serious consideration of the value of partial salpingectomy. The procedure may not confer the benefit of avoiding recurrent ectopic, and could in fact confuse the picture, as ectopic would be ruled out on the basis of prior salpingectomy, as was the case here. The message from this case is that salpingectomy for the management of ectopic pregnancy or its prevention must be complete. This is important even when assisted reproductive techniques are being used [7]. Total salpingectomy should be straight forward during laparotomy.

Laparoscopically, some may find it difficult to place the endo-loops at the cornu, though this can be achieved with experience by ensuring proper positioning of the plastic rod before tightening the loop. The procedure should be a lot easier with diathermy (uniploar and bipolar), harmonic scalpel as well as plasmakinetic instruments.

\section{References}

1. Cheong Y, Li TC (2007) Controversies in the management of ectopic pregnancy. Reprod Biomed Online 15:396-402

2. Yao M, Tulandi T (1997) Current status of surgical and nonsurgical management of ectopic pregnancy. Fertil Steril 67:421-433

3. Glock JL, Loy RA, Brumsted JR (1993) Reproductive performance following a second ectopic gestation. Int $\mathrm{J}$ Gynaecol Obstet 43:191-194

4. Rizos A, Eyong E, Yassin A (2003) Recurrent ectopic pregnancy at the ipsilateral fallopian tube following laparoscopic partial salpingectomy, with endo-loop ligation. J Obstet Gynaecol 23:678-679

5. Zurarte R, Khong CC (2005) Recurrent ectopic pregnancy following ipsilateral partial salpingectomy. Singap Med J 46:476478

6. Adair CD, Benrubi GI, Sanchez-Ramos L, Rhatigan R (1994) Bilateral tubal ectopic pregnancies after bilateral partial salpingectomy. A case report. J Reprod Med 39:131-133

7. Dubuisson JB, Aubriot FX, Mathieu L, Foulot H, Mandelbrot L, de Joliere JB (1991) Risk factors for ectopic pregnancy in 556 pregnancies after in vitro fertilization: implications for preventive management. Fertil Steril 56:686-690 